\title{
Noninvasive ventilation in acute exacerbation of COPD: Homer is close to shore
}

\author{
Umur Hatipoğlu, MD, MBA' ${ }^{1}$ Laith Ghazala, MD ${ }^{1,2}$, Manshi Li, MS ${ }^{3}$, Xiaofeng Wang, PhD ${ }^{3}$, Abhijit Duggal, MD, MPH
}

U Hatipoğlu, L Ghazala, M Li, X Wang, A Duggal. Noninvasive ventilation in acute exacerbation of COPD: Homer is close to shore. Can J Respir Ther 2021;57:160. doi: 10.29390/cjrt-2021-072.

Key Words: COPD; acute hypercapnic respiratory failure; noninvasive ventilation; outcome prediction

\section{Dear Editor,}

We thank Dr. Karim and colleagues for their interest in our paper [1]. As they point out in their Letter to the Editor [2], retrospective studies are hypothesis generating rather than conclusive. When biologically plausible variables are linked to outcomes in a multivariable regression analysis, the urge to claim a cause-and-effect relationship is great but should be resisted. We believe that we made this point clear throughout our manuscript.

Our study found that noninvasive ventilation (NIV) prevents invasive mechanical ventilation in most patients with severe hypercapnic respiratory failure due to Chronic Obstructive Pulmonary Disease (COPD). We found that the best predictive model for NIV failure included two independent variables: body mass index (BMI) and Acute Physiology and Chronic Health Evaluation (APACHE) III. The latter should not come as a surprise since APACHE III score includes all elements of HACOR score, a validated index for predicting NIV failure in COPD [3]. Inclusion of the BMI improved model discrimination; however, we should note that BMI was not different between the failure and success groups $(P=0.075)$. The instance when a nonsignificant variable by classical significance threshold could improve model prediction is not uncommon.

As alluded to in our discussion, the jury is still out on the association of BMI on NIV failure with some studies showing no effect [4] and others a salutary one in the outpatient setting [5]. Again, when building a predictive model, this issue is moot since proof of a causal relationship is not the goal.

We found that duration of NIV was not different between the failure and success groups (2-sample t-test). Further, in a multivariable regression analysis performed using the entire cohort, duration of application did not contribute to the predictive ability of the model. We would agree that this finding is not conclusive. A true difference may not have been uncovered due to inadequate power. Absence of the difference could be related to the setting in which the study was conducted (a quaternary care center with multidisciplinary teams and high monitoring capability). However, the likelihood that duration of NIV plays an important role in failure and success should be low given that more than $80 \%$ of the binary NIV outcome could be explained by severity of illness and BMI. Recent reports on the use of NIV among patients with COVID-19 pneumonia seem to support this notion. Papoutsi and colleagues [6] performed a meta-analysis of 12 studies that included 8944 critically ill patients and found no difference in mortality among patients who underwent early versus late intubation. Their findings extended to the setting of a prior trial of noninvasive mechanical ventilation [6]. Unfortunately, our database did not include obstructive sleep apnea and therefore we could not evaluate patients with overlap syndrome separately. We concur that this would have been of interest.

Indeed, the saga continues but we feel that Homer's ships are near the shore. We can see clearly that NIV can save lives and time on invasive mechanical ventilation for patients with COPD who have acute hypercapnic respiratory failure. With careful monitoring of adverse effects and attention to clinical signs of failure, we think the duration of NIV is unlikely to be a deciding variable for outcomes.

\section{REFERENCES}

1. Ghazala L, Hatipoğlu U, Devnani T, et al. Duration of noninvasive ventilation is not a predictor of clinical outcomes in patients with acute exacerbation of COPD and respiratory failure. Can J Respir Ther 2021;57:113-8. doi: 10.29390/cjrt-2021-021.

2. Karim HMR, Araújo MJ, Esquinas AM. Noninvasive ventilation duration as an outcome predictor in acute exacerbation of COPD and respiratory failure: the saga continues. Can J Respir Ther 2021;57:147. doi: 10.29390/cjrt-2021-069.

3. Duan J, Wang S, Liu P, et al. Early prediction of noninvasive ventilation failure in COPD patients: derivation, internal validation, and external validation of a simple risk score. Ann Intensive Care 2019;9(1):108. doi: 10.1186/s13613-019-0585-9.

4. Steriade AT, Johari S, Sargarovschi N, et al. Predictors of outcome of noninvasive ventilation in severe COPD exacerbation. BMC Pulm Med 2019;19(1):131. doi: 10.1186/s12890-019-0892-9.

5. Altinoz H, Adiguzel N, Salturk C, et al. Obesity might be a good prognosis factor for COPD patients using domiciliary noninvasive mechanical ventilation. Int J Chron Obstruct Pulmon Dis 2016;11:1895-901. doi: 10.2147/COPD.S108813.

6. Papoutsi E, Giannakoulis VG, Xourgia E, Routsi C, Kotanidou A, Siempos II. Effect of timing of intubation on clinical outcomes of critically ill patients with COVID-19: a systematic review and meta-analysis of non-randomized cohort studies. Crit Care 2021;25(1):121. doi: 10.1186/s13054-021-03540-6.

\footnotetext{
${ }^{1}$ Respiratory Institute, Cleveland Clinic, Cleveland, OH, USA

${ }^{2}$ Trillium Health Partners, Mississauga, ON, Canada

${ }^{3}$ Quantitative Health Sciences, Cleveland Clinic, Cleveland, OH, USA

Correspondence: Laith Ghazala, E-mail: laithgazala@gmail.com
}

Published online at https://www.cjrt.ca on 29 November 2021 